PWE-12 PSYCHOSOCIAL RISK FACTORS ARE SIGNIFICANTLY ASSOCIATED WITH HIGHER MORTALITY IN PATIENTS UNDERGOING LIVER TRANSPLANTATION

Jeevan Barn, J Barn*, L Downey, A Wetten, L MacDougall, M Hudson. Liver Unit, The Newcastle upon Tyne Hospitals NHS Foundation Trust, Newcastle upon Tyne, UK

\subsection{6/gutjnl-2021-BSG.202}

Introduction Transplantation is an established therapy for endstage liver disease and there is great demand for donor organs. Psychosocial concerns are prevalent in patients undergoing liver transplant (OLT) assessment and this may impact on post-transplant outcomes. In the UK there is no current standardised method quantifying OLT candidates' psychosocial risk. We investigated the use of a psychosocial risk assessment tool in assessing post-transplantation outcomes.

Methods A psychosocial risk profile tool (mSIPAT) was retrospectively applied to all patients who underwent elective OLT assessment between January 2010 and December 2014. Electronic records were reviewed from the date of assessment and data was collected regarding assessment outcome, transplantation, late onset medical/psychiatric complications and mortality. Results 273 patients were identified during the 5-year time period. The median age of patients at the time of assessment was 57 years and $62.3 \%$ of patients were male. Of those assessed 168 patients were listed for transplant and 126 underwent OLT. The main indication for transplant was synthetic dysfunction (35.9\%). 178 (65\%) patients assessed were classed as meeting criteria for medium/high psychosocial risk (MPSR). MPSR patients when compared to low-risk patients (LPSR) were more likely to have an underlying alcohol related liver disease $(p=0.005)$, were less likely to undergo transplantation $(\mathrm{p}<0.001)$ and were more likely to return to alcohol misuse post assessment $(\mathrm{p}<0.001)$. MPSR patients had a significantly higher all-cause mortality than LPSR patients $(p=0.005)$. Patients who died scored significantly higher in the majority of the components of the mSIPAT. Undergoing transplantation (OR $0.10 \quad(95 \% \mathrm{CI} \quad 0.06-0.20)$ ) and concerns regarding financial support (OR 4.85 (95\%CI 1.16-20.32) were factors independently associated with mortality when corrected for age and gender. Figure 1 illustrates the survival

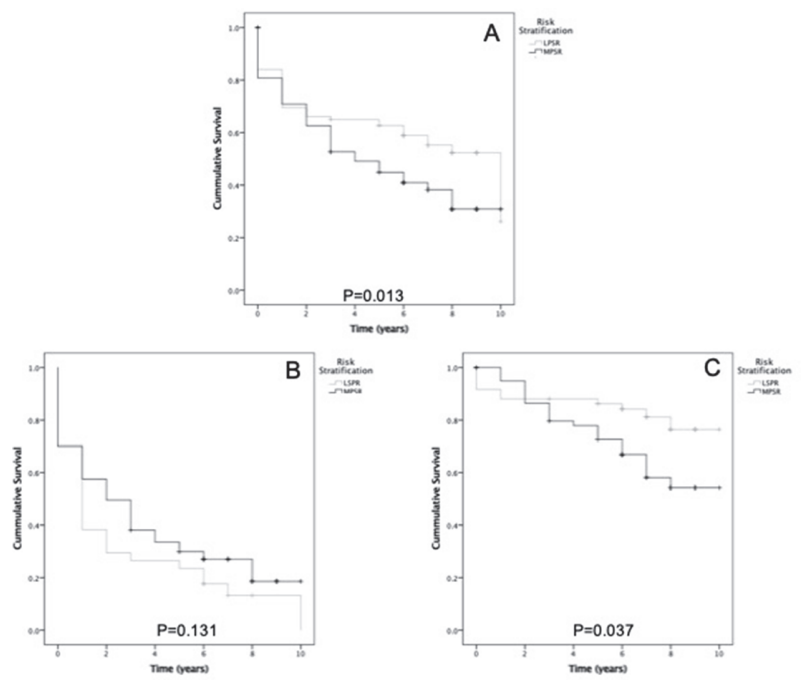

Abstract PWE-12 Figure 1 Kaplan-Meier survival curves comparing risk stratification groups in a) overall patient cohort, b) Patients who did not undergo transplantation and c) patients who were transplanted difference between those who classed as MPSR and LPSR. In those transplanted MPSR patients had a significantly higher mortality $(p=0.037) .35(27.7 \%)$ patients who underwent transplant died during the follow up period. Factors significantly associated with mortality post transplantation were recidivism $(p=0.026)$ and concerns regarding social $(p=0.002)$ and financial support $(\mathrm{p}=0.050)$.

Conclusions Patients classed as MSPR had a higher all-cause mortality and an increased likelihood of recidivism. Using the mSiPAT as part of the elective OLT assessment can potentially identify both those at highest risk and areas on which to focus support in order to prevent poor outcomes.

\section{PWE-13 EXPERIENCE OF SHIELDING DURING FIRST WAVE OF COVID-19 AMONGST UK PATIENTS WITH AUTOIMMUNE HEPATITIS (AIH)}

Victoria Appleby*, Chenchu R Chimakurthi, Ceri Watson, Stewart Gibson, Vinod Hegade, Udvitha Nandasoma, Matthew Huggett, Rebecca L Jones. The Leeds Liver Unit, Leeds, UK

\subsection{6/gutjnl-2021-BSG.203}

Introduction In March 2020, severe acute respiratory syndrome SARS-CoV-2 was declared a pandemic. Specific shielding guidance produced by PHE aimed to protect clinically extremely vulnerable individuals. This included people with $\mathrm{AIH}$ on immunosuppressants. In the first wave, advice included not leaving home. The practicalities of this and impact of enforced shielding on mental health (MH) and well-being is not well documented, although suicide theories emphasise the negative effect of social isolation and loneliness. We conducted a patient survey to assess the impact of shielding in our AIH cohort.

Methods Between 1Jul -10 Aug 2020,292 patients with AIH, fulfilling shielding criteria were invited to participate in a clinician designed survey on MSForms ${ }^{\circledR}$ online or by hand. Paper responses were entered online for analysis.

Results 36\%(104)participated;79\% female,74\% age 25-69. Immunosuppression burden:50\% single agent,47\%dual, 3\% on $\geq 3$.Shielding compliance was ranked on a scale from 0 10:60\% 9-10/10,29\%7-8/10,11\%0-6/10. Reasons for breaches included: exercise, shopping, to have a drive. Responses demonstrated assessment of risk by those breaching guidance. Ease with practical aspects of living were assessed on a scale from 0-10. One third found this 'very easy',1/3 'quite easy',1/3 'not very easy'. For shopping, respondents used family members(49\%), online(39\%), shop in person(8\%), other(4\%).NHS volunteers were not used for shopping. Obtaining prescriptions was reported as easy in 79\%: 76\% used family/online pharmacy deliveries, other methods included: friend/neighbour, hospital delivery, NHS volunteer, collection in person. Of those with long-term partners $95 \%$ continued co-habiting but $4 \%$ lived separately in this period, remaining respondents previously lived alone. Respondents were asked to rank their $\mathrm{MH}$ and physical well-being. $27 \%$ reported $\mathrm{MH}$ as 'high', 27\%'moderate',46\%'low'. Whilst $87 \%$ had access to outdoor space, only $46 \%$ had access to space large enough to exercise. Questions on the impact of shielding on other household members highlighted the perceived loss of independence and 'guilt' associated with the negative impact of shielding on others. Pertaining to work/employment, 38\%(40/104) were employed in March 2020. Of those answering the question 
'How did you work during the shielding period': 54\% worked from home (WFH), prior to COVID-19 only 5\% WFH. 2\% continued to commute to work.

Conclusion This survey has provided a unique insight into shielding attitudes and behaviours in patients with AIH during the pandemic first wave demonstrating its negative impact on $\mathrm{MH}$ and well-being. As further evidence emerges regarding the risk of COVID-19 in adults taking immunosuppressants, more detailed risk stratification may be possible; this would be advantageous from a patient perspective.

\section{PWE-14 END OF LIFE CARE IN PATIENTS WITH CIRRHOSIS: A DISTRICT GENERAL HOSPITAL PERSPECTIVE}

Emma Saunsbury*, Daniel Maggs. Royal United Hospitals NHS Foundation Trust, Bath, UK

\subsection{6/gutjnl-2021-BSG.204}

Introduction End of life care (EOLC) refers to care given to patients with a prognosis of $<1$ year. Whilst cirrhosis-related death can be unforeseen, it typically concludes a prolonged declining clinical trajectory. Clinical encounters therefore represent key opportunities for EOLC planning in this cohort, yet the limitations of current provision by gastroenterologists are increasingly recognised. ${ }^{1}$ We reviewed our practice to identify areas for improvement.

Methods This retrospective cohort study identified patients who died from sequelae of cirrhosis between January 2018December 2019 (i.e. pre-COVID) and had >1 non-terminal cirrhosis-related admission in their last year of life. Electronic records were interrogated for evidence of prognostication assessment, transplant candidacy and gastroenterology input. Discussions regarding end-stage liver disease (ESLD), EOLC and palliative care referral were reviewed.

Results 52 patients were identified for analysis. In their last year of life, patients averaged 1.7 cirrhosis-related admissions and $69.2 \%$ had $>1$ outpatient clinic. $61.5 \%$ had no prognostication score documented, including $58.3 \%$ (7/12) of ChildPugh C patients. Interestingly, only $23.6 \%$ met $>3$ poor-prognosis criteria prior to their terminal admission. ${ }^{1}$

ESLD was discussed in a quarter of patients in advance of terminal admission, yet EOLC was subsequently broached in only $61.5 \%(8 / 13)$ of these cases. Just $33.3 \%$ of Child-Pugh $\mathrm{C}$ patients and $65.3 \%(17 / 26)$ of non-transplantable patients were counselled regarding ESLD. In terms of palliative care provision, $19.2 \%(10 / 52)$ received inpatient palliative care, though $80 \%$ of referrals only occurred during their terminal admission. $62.5 \%$ of community palliative care referrals occurred in the context of a Continuing Healthcare Fast Track discharge.

Conclusions We have highlighted that more needs to be done to resolve the gaps in our patient pathway to ensure ESLD is recognised, patients are counselled appropriately and fitting EOLC is offered. Enhanced EOLC planning in this high-risk group is a priority, as currently palliative care input is mostly limited to a late stage in the patient journey.

Validated prognostication tools can identify those with poorer prognoses yet, without a formal pathway to review this, patients with declining trajectories were not readily recognised. We plan to introduce an inpatient 'discharge bundle' and liver MDT to prompt enhanced follow up of ESLD patients. The goal is to achieve an optimised parallel care model, with earlier EOLC offered alongside ongoing active management in this cohort.

\section{REFERENCE}

1. Hudson BE, Ameneshoa K, Gopfert A et al. Integration of palliative and supportive care in the management of advanced liver disease: development and evaluation of a prognostic screening tool and supportive care intervention. Frontline Gastroenterol 2017; 8(1):45-52

\section{PWE-15 IGG4 RELATED HEPATOBILIARY DISEASE; EXPERIENCE IN A TERTIARY CENTRE}

Wafaa Ahmed*, Baljinder Sidhu, Devinder Bansi, Panagiotis Vlavianos, Christopher Wadsworth, Shwan Karim, Natalie Phillips. Imperial College Healthcare NHS Trust, London, UK

\subsection{6/gutjnl-2021-BSG.205}

Introduction IGG4 related hepatobiliary disease (IGG4-HBD) is part of a multisystem fibroinflammatory disorder that can present with abnormal liver function tests alongside symptomatic biliary obstruction, cholangiopathy and pseudo-tumours. We report on the clinical course of a cohort of patients with IGG4HBD managed in a tertiary hepatobiliary centre.

Methods Consecutive patients with IGG4-HBD over a five year period were retrospectively identified. Patients were diagnosed based on histology, imaging and serum IGG4 levels. Recorded variables included patient demographics, endoscopic procedures and response to therapy.

Results

Seventeen patients were identified The median age at diagnosis was 60 years old (range 28-85 years old). Six (35.3\%) presented as a possible malignancy, with pseudo-tumours found in four $(23.5 \%)$. Four $(23.5 \%)$ patients had other sites of IGG4 involvement outside the hepatobiliary system. Two cases had established cirrhosis and one had established chronic pancreatitis at diagnosis.

Histology was obtained via Endoscopic Ultrasound (EUS) in $12(70.6 \%)$ patients (one false negative), three (17.6\%) via Endoscopic Retrograde Cholangio-pancreatography (ERCP) with biopsies obtained from within the bile duct and two $(11.8 \%)$ had transcutaneous biopsies. Median IGG4 levels at diagnosis was $5.55 \mathrm{~g} / \mathrm{L}$ (range $0.73-12.48 \mathrm{~g} / \mathrm{L}$ ). ERCP was performed on $13(76.5 \%)$ patients prior to induction therapy to relieve biliary obstruction. A straight plastic stent was inserted in one case with the rest having a fully covered selfexpanding metal stent (FC-SEMS).

Fifteen (88.2\%) patients received Prednisolone for induction. Prednisolone monotherapy was used for maintenance in $8(47.1 \%)$ patients and the same number had dual therapy with Azathioprine. Rituximab was utilized in 3 (17.6\%) patients. All of which had been on Prednisolone for a minimum of 2 years and Azathioprine (6 weeks, 2 months and 11 years) before stopping due to severe side effects with subsequent relapse of their IGG4-HBD.

Four $(23.5 \%)$ were not on maintenance immunosuppression and two (15.4\%) patients had stricture resolution with placement of FC-SEMS alone. Patients have been under follow up for a median of 37 months (range 8-144 months). All had marked improvement in biliary strictures following treatment and are currently in remission.

Conclusions We describe a cohort of patient with IGG4 HBD. We show that in this setting EUS guided biopsy alongside ERCP is an effective method of tissue acquisition and that ERCP and biliary drainage can be used as an adjunct to 